\title{
New combinations in Senegalia and Vachellia (Leguminosae: Mimosoideae) for Southeast Asia and China
}

\author{
B.R. Maslin ${ }^{1}$, D.S. Seigler ${ }^{2}$, J. Ebinger ${ }^{3}$
}

Key words

Acacia

China

Leguminosae

Mimosoideae

SE Asia

Senegalia

Vachellia

\begin{abstract}
Fragmentation of the former broadly circumscribed genus Acacia (Leguminosae: Mimosoideae) necessitates combinations in Senegalia (syn. Acacia subg. Aculeiferum) and Vachellia (syn. former Acacia subg. Acacia) for the following 40 taxa that are recorded for the region encompassed by Southeast Asia and China. Senegalia andamanica, S. borneensis, S. caesia, S. comosa, S. delavayi, S. delavayi var. kunmingensis, S. donnaiensis, S. gageana, S. intsia, S. kekapur, S. kostermansii, S. meeboldii, S. megaladena, S. megaladena var. garrettii, S. megaladena var. indochinensis, S. merrillii, S. palawanensis, S. pennata subsp. hainanensis, S. pennata subsp. insuavis, S. pluricapitata, S. pluriglandulosa, S. pruinescens, S. pseudointsia, S. sulitii, S. tawitawiensis, S. teniana, S. thailandica, S. tonkinensis, S. torta, S. verheijenii, S. vietnamensis and S. yunnanensis. Vachellia harmandiana, V. inopinata, V. kingii, V. leucophloea, V. leucophloea var. microcephala, V. myaingii, V. siamensis (syn. Acacia craibii) and $V$. tomentosa. Senegalia rugata replaces the well-known name Acacia concinna. There are four doubtful names for the region, namely, Acacia brunnescens, A. microcephala, A. obcordata and A. pseudoarabica.
\end{abstract}

Published on 26 June 2013

\section{INTRODUCTION}

Molecular and other evidence has shown that the former, broadly circumscribed genus Acacia Mill. (Leguminosae: Mimosoideae) is polyphyletic; it is now regarded as comprising at least five genera, Acacia (s.str.), Acaciella Britton \& Rose, Mariosousa Seigler \& Ebinger, Senegalia Raf. and Vachellia Wight \& Arn. (see Miller \& Seigler 2012 for the overview of the new generic classification). Collectively, these genera are called Acacia s.lat. in the discussion below. Acacia is now conserved with a new type (see McNeill \& Turland 2011), requiring new names in Vachellia for many taxa formerly included in Acacia subg. Acacia; new names are also needed for many taxa of the segregate genus Senegalia (syn. Acacia subg. Aculeiferum Vassal). Both these genera are pantropical.

Names are already available for all New World and Australian taxa of Senegalia and Vachellia, and for most taxa in the Africa/ Asia region (see Seigler \& Ebinger 2005, 2010, Glass \& Seigler 2006, Kodela \& Wilson 2006, Banfi \& Galasso 2008, Hurter \& Mabberley in Mabberley 2008, Clarke et al. 2009, Maslin 2012, Kyalangalilwa et al. 2013). Therefore, apart from Madagascar which has very few species, Asia and the Middle East are the only regions for which combinations are needed in order that all taxa of Acacia s.lat. will have names available under the new classification.

The present paper provides combinations for the indigenous taxa of Southeast Asia and China that are referable to Senegalia and Vachellia. This is not a taxonomic revision of these two genera. In undertaking this work we have relied on previous

\footnotetext{
Western Australian Herbarium, Department of Environment and Conservation, Locked Bag 104, Bentley Delivery Centre, Western Australia 6983; corresponding author e-mail: bruce.maslin@dec.wa.gov.au.

2 Department of Plant Biology, University of Illinois, Urbana, Illinois 61801, USA.

${ }^{3}$ Emeritus Professor of Botany, Eastern Illinois University, Charleston, Illinois 61920, USA.
}

relevant studies of Acacia s.lat., the most important being those of the late Ivan Nielsen (Nielsen 1980, 1981, 1985a, b and 1992) for Southeast Asian taxa and Wu De-Lin \& Nielsen (2010) for those from China. Unless otherwise indicated, all new combinations presented here apply to taxa that were accepted as current under Acacia in these publications. Full synonymy (under Acacia) for these taxa is given in the above references, and in Roskov et al. (2005, which extensively cites the works of Nielsen). The only synonyms included here are basionyms and names that were accepted as current under Acacia in the above works. We have also noted cases where taxonomic opinion differs from that which we have adopted.

Apart from the 40 new combinations presented here, the only indigenous taxa of Vachellia and Senegalia in Southeast Asia and China are the following: S. catechu (L.f.) P.J.H.Hurter \& Mabb., S. chundra (Roxb. ex Rottler) Maslin, S. ferruginea (DC.) Pedley, S. pennata, S. pennata subsp. kerrii (I.C.Nielsen) Maslin, $S$. rugata (see below under this species for discussion) and V. nilotica subsp. indica (Benth.) Kyal. \& Boatwr. and S. brevispica (Harms) Seigler \& Ebinger subsp. brevispica (see Kyalangalilwa et al. 2013).

Unless otherwise indicated, type information for most taxa has been taken from Nielsen (1980, 1981, 1985a); in the remaining cases the type citation from the protologue is provided. Type specimens have not been checked by the authors. Distributions given below are taken from Roskov et al. (2005) and Wu De-Lin \& Nielsen (2010) unless otherwise stated.

The geographic scope of this paper includes Southeast Asia and southern China. As used here the term Southeast Asia is the area extending from Myanmar (Burma), Vietnam, Laos, Cambodia and Thailand to the Malesian region as defined by Van Steenis (1948). 


\section{NEW COMBINATIONS}

\section{Senegalia Raf.}

(syn. Acacia subg. Aculeiferum Vassal)

1. Senegalia andamanica (I.C.Nielsen) Maslin, Seigler \& Ebinger, comb. nov.

Acacia andamanica I.C.Nielsen, Adansonia sér. 2, 19 (1980) 354. - Type: King's Collector s.n. (lecto K, Nielsen 1980: 354), Andaman Isl.

Distribution — India (Andaman Islands), Thailand.

2. Senegalia borneensis (I.C.Nielsen) Maslin, Seigler \& Ebinger, comb. nov.

Acacia borneensis I.C.Nielsen, Opera Bot. 81 (1985) 20, f. 9 (7-13). — Type: Endert 2189 (holo L; iso A, K), Borneo, C.E. Kalimantan, W. Kutai, m. 10/11 near Mt Antjaloeng, 15 m, 24 July 1925.

Distribution — Indonesia (Kalimantan), Malaysia (Sabah).

3. Senegalia caesia (L.) Maslin, Seigler \& Ebinger, comb. nov.

Mimosa caesia L., Sp. PI. ed.1, 1 (1753) 522. - Acacia caesia (L.) Willd. (1806) 1090. - Lectotype: Herb. Herman 2: 50, No. 217, BM (Rico in Turland \& Jarvis 1997: 475)

Distribution — India, Sri Lanka, Bhutan, Bangladesh, Myanmar (Burma), central and southern China (Guangdong, Hainan, Sichuan \& Yunnan Provinces), Thailand, Laos, Cambodia, Vietnam, Taiwan.

Notes - Although A. caesia var. subnuda is recognized by Roskov et al. (2005), based on the works of Nielsen (1981: 53) and Sanjappa (1992: 37), it is regarded as conspecific with A. caesia by Chakrabarty \& Gangopadhyay (1996: 604) and Wu De-Lin \& Nielsen (2010: 57). We have therefore followed the most recent taxonomy in not recognizing the variety here. However, it should be noted that Nielsen should have adopted the name var. oxyphylla for this taxon; therefore, if this entity is ever recognized as a variety, either in Acacia or Senegalia, then the combination based on $A$. oxyphylla will need to be made.

Although Roskov et al. (2005) list $A$. caesia as occurring in Java and the Philippines it is doubtfully native there because it is not listed for these regions by Nielsen (1992) or Wu De-Lin \& Nielsen (2010).

4. Senegalia comosa (Gagnep.) Maslin, Seigler \& Ebinger, comb. nov.

Acacia comosa Gagnep., Notul. Syst. (Paris) 2 (1911) 113. - Type: Pierre 5977 (lecto P; iso K, L, Nielsen 1980: 348), South Vietnam, Bien Hoa, Mar. 1869.

Distribution — Thailand, Laos, Cambodia, Vietnam (southern).

\section{Senegalia delavayi (Franch.) Maslin, Seigler \& Ebinger,} comb. nov.

Acacia delavayi Franch., PI. Delavay. (1890) 194. - Type citation: Delavay, "Yun-nan, in collibus incultis ad Kiang-yn; fruct. 5. Sept. 1888".

Distribution - China (Guizhou, Yunnan).

Note - Varieties are not given in Roskov et al. (2005) but they are recognized in Wu De-Lin \& Nielsen (2010) whose classification is adopted here.

\section{a. var. delavayi}

Acacia delavayi C.Chen \& H.Sun var. delavayi (Sun \& Chen 1990: 261).

Distribution — China (Yunnan) b. var. kunmingensis (C.Chen \& H.Sun) Maslin, Seigler \& Ebinger, comb. nov.

Acacia delavayi var. kunmingensis C.Chen \& H.Sun, Acta Bot. Yunnan. 12 (1990) 262. - Type: Xiangkun Deng 673 (holo KUN), Kunming (translation of Chinese script by Libing Zhang).

Distribution - China (Guizhou, Yunnan).

6. Senegalia donnaiensis (Gagnep.) Maslin, Seigler \& Ebinger, comb. nov.

Acacia donnaiensis Gagnep., Not. Syst. (Paris) 2 (1911) 114. - Type: Harmand 965 (lecto P, Nielsen 1980: 349), Vietnam, bords du Donnai, Nov. 1876

Distribution - Cambodia, Vietnam (southern), Malaysia (Sabah), Indonesia (Kalimantan).

7. Senegalia gageana (Craib) Maslin, Seigler \& Ebinger, comb. nov.

Acacia gageana Craib, Bull. Misc. Inform. Kew (1915) 409. - Type: J.D. Hooker s.n. (holo K; iso CAL, Chakrabarty \& Gangopadhyay 1996: 604), India, Sikkim.

Distribution - Pakistan, Nepal, Bhutan, India, Bangladesh; also recorded for Myanmar (Burma) by Kress et al. (2003: 299).

Note - Sanjappa (1992: 40), Kumar \& Sane (2003: 86), Kress et al. (2003) and Roskov et al. (2005) recognize this entity as a distinct species, but Chakrabarty \& Gangopadhyay (1996: $604,606)$ consider it conspecific with $A$. (Senegalia) caesia.

8. Senegalia intsia (L.) Maslin, Seigler \& Ebinger, comb. nov. Mimosa intsia L., Sp. PI. (1753) 522. - Lectotype: 'Inja' in Rheede, Hort. Malab. 6 (1686) t. 4 [icon], (Rico in Turland \& Jarvis 1997: 476).

Distribution - Nepal, India, Sri Lanka, Bangladesh, Myanmar (Burma).

Note - Acacia intsia is recognized as a distinct species by Sanjappa (1992: 40), Kumar \& Sane (2003: 87) and Roskov et al. (2005). However, Chakrabarty \& Gangopadhyay (1996: 606) consider this a nomen confusum and refer the Indian material of it to A. caesia.

9. Senegalia kekapur (I.C.Nielsen) Maslin, Seigler \& Ebinger, comb. nov.

Acacia kekapur I.C.Nielsen, Opera Bot. 81 (1985) 13, f. 5 (1-8). - Type Winckel 438B (holo L; iso BO), Java, Preanger, $\mathrm{S}$ of Tjibeber, Tjadas Malang, $\pm 1000 \mathrm{~m}, 20$ Oct. 1919 .

Distribution — Indonesia (Sumatra, Java), Singapore.

10. Senegalia kostermansii (I.C.Nielsen) Maslin, Seigler \& Ebinger, comb. nov.

Acacia kostermansii I.C.Nielsen, Opera Bot. 81 (1985) 15, f. 5 (9-14). Type: Elbert 4140 (holo BO; iso L), Lesser Sunda Islands, Sumbawa, Sultanat Sumbawa, bei Lapi, 100-200 m, 1910.

Distribution — Indonesia (Lesser Sunda Islands: Sumbawa, West Flores, Alor)

11. Senegalia meeboldii (Craib) Maslin, Seigler \& Ebinger, comb. nov.

Acacia meeboldii Craib, Bull. Misc. Inform. Kew (1928) 66. - Type: Kerr 12197 (lecto ABD; iso BM, K; Nielsen 1980: 350), Thailand, Chumphon, Lang Suan, Pato, $200 \mathrm{~m}$, common in evergreen forest.

Distribution — Myanmar (Burma), Thailand 
12. Senegalia megaladena (Desv.) Maslin, Seigler \& Ebinger, comb. nov.

Acacia megaladena Desv., J. Bot. Agric. 3 (1814) 69. - Type: Desvaux s.n. (holo P, Nielsen 1980: 350), Inde orientale.

Distribution - Nepal, India, Bangladesh, Myanmar (Burma), China (Guangxi, Yunnan), Thailand, Laos, Cambodia, Vietnam, Malaysia (northern Peninsular Malaysia), Indonesia (Java), Philippines.

\section{a. var. megaladena}

Acacia megaladena var. megaladena (Nielsen 1980: 351).

Distribution - Nepal, India, Myanmar (Burma), China (Guangxi ,Yunnan), Thailand, Laos, Vietnam (northern), Indonesia (Java).

b. var. garrettii (I.C.Nielsen) Maslin, Seigler \& Ebinger, comb. nov.

Acacia megaladena var. garrettii I.C.Nielsen, Adansonia sér. 2, 19 (1980) 351. - Type: Garrett 1239 (holo K; iso ABD, E), Thailand, Chiang Mai, Doi Chawm Hot, c. 1420 m.

Distribution - China (Guangxi, Yunnan), Thailand.

c. var. indochinensis (I.C.Nielsen) Maslin, Seigler \& Ebinger, comb. nov.

Acacia megaladena var. indochinensis I.C.Nielsen, Adansonia sér. 2, 19 (1980) 351. - Type: Larsen, Smitinand \& Warncke 375 (holo AAU), South-eastern Thailand, Prachin Buri, KhaoYai National Park, alt. 750 m.

Distribution - Cambodia, Thailand, Laos, Vietnam (southern), Malaysia (northern Peninsula Malaysia, but not listed for this region in Turner (1997: 290), Indonesia (Java).

13. Senegalia merrillii (I.C.Nielsen) Maslin, Seigler \& Ebinger, comb. nov.

Acacia merrillii I.C.Nielsen, Opera Bot. 81 (1985) 9, f. 2. - Type: Conklin PNH 18680 (holo P; iso A, L), Philippines, Mindoro, Mt Yagaw, SW slope, Lumun, alt. 330 m, 27 July 1953.

Distribution - Indonesia (Sulawesi, Moluccas - Mangole Island \& Halmahera Island), Philippines.

14. Senegalia palawanensis (I.C.Nielsen) Maslin, Seigler \& Ebinger, comb. nov.

Acacia palawanensis I.C.Nielsen, Opera Bot. 81 (1985) 16, f. 6 (7-11). Type: Sulit PNH 12528 (holo L; iso A, US), Philippines, Palawan, Mt Iraan, SE Slope, Aborlan, vic. of Ibato River, 100 m, 5 June 1950.

Distribution - Philippines (Palawan).

\section{Senegalia pennata (L.) Maslin}

Senegalia pennata (L.) Maslin (2012) 466.

This species comprises three subspecies but the combination Senegalia pennata subsp. kerrii has already been provided (Maslin 2012).

a. subsp. hainanensis (Hayata) Maslin, Seigler \& Ebinger, comb. nov.

Acacia hainanensis Hayata, Ic. PI. Formos. 3 (1913) 86. - Acacia pennata subsp. hainanensis (Hayata) I.C.Nielsen (1980) 352. - Type: Katzumada s.n. (holo TI), Hainan, 1910.

Distribution - India, Myanmar (Burma), China (Fujian, Guangdong, Guangxi, Hainan, Yunnan), Vietnam. b. subsp. insuavis (Lace) Maslin, Seigler \& Ebinger, comb. nov.

Acacia insuavis Lace, Bull. Misc. Inform. Kew (1915) 401. - Acacia pennata subsp. insuavis (Lace) I.C.Nielsen (1980) 353. - Type: Lace 6173 (holo $\mathrm{E}$; iso K), Burma.

Distribution — India, Myanmar (Burma); uncertain for Thailand, Laos, Cambodia.

16. Senegalia pluricapitata (Steud. ex Benth.) Maslin, Seigler \& Ebinger, comb. nov.

Acacia pluricapitata Steud. ex Benth., London J. Bot. 1 (1842) 516. — Type: G. Porter in Wallich Cat. no. 5255A (holo K-WALL)

Distribution - Burma, Thailand, Vietnam (southern), Malaysia (Peninsular Malaysia), Indonesia (Sumatra, Java, Kalimantan), Philippines.

17. Senegalia pluriglandulosa (Verdc.) Maslin, Seigler \& Ebinger, comb. nov.

Acacia pluriglandulosa Verdc., Kew Bull. 32 (1978) 472. — Type: Hoogland \& Craven 10334 (holo K; iso A, BO, BRI, CANB, L, LAE, US), Papua New Guinea.

Distribution — Philippines, Indonesia (Papua), Papua New Guinea.

18. Senegalia pruinescens (Kurz) Maslin, Seigler \& Ebinger, comb. nov.

Acacia pruinescens Kurz, J. Asiat. Soc. Bengal, Pt. 2, Nat. Hist. 45 (1877) 296, 298. - Type: J.D.Anderson s.n. (lecto CAL, Nielsen 1980: 355), Burma (Poneshee district), 26 April 1866.

Distribution — India, Myanmar (Burma), China (Guangxi, Yunnan), Vietnam (northern).

19. Senegalia pseudointsia (Miq.) Maslin, Seigler \& Ebinger, comb. nov.

Acacia pseudointsia Miq., FI. Ned. Ind. 1 (1855) 12, as 'Pseudo-Intsia'. Type: Blume s.n. (holo L), Java.

Distribution - Thailand, Malaysia (Peninsula, Sabah, Sarawak), Indonesia (Sumatra, Java), Singapore.

Note - In accordance with Art. 60.9 of the ICN (McNeill et al. 2012) the hyphen is removed from this name ('pseudo-intsia' has commonly been used in literature, including Roskov et al. 2005).

\section{Senegalia rugata (Lam.) Britton \& Rose}

Senegalia rugata (Lam.) Britton \& Rose (1928) 120. - Mimosa rugata Lam., Encycl. 1 (1783) 20. - Type: Sonnerat s.n. (holo P-LAM), India. Mimosa concinna Willd., Sp. PI. ed. 4, 4 (2) (1806) 1039. — Acacia concinna (Willd.) DC. (1825) 464. - Type: D. Klein s.n. (holo B-WILLD), Ind. Or. Acacia rugata Buch.-Ham. ex Benth. (1842) 514. - Type: Wallich Cat. no. 5251 (holo K-WALL).

Distribution - India, Nepal, Bhutan, Bangladesh, Myanmar (Burma), Laos, Cambodia, Thailand, Vietnam, Malaysia (Peninsula), Indonesia (Sumatra, Java, Sumba, Flores, Sulawesi, Ambon, Moluccas, Kai Is.), Singapore, Philippines, Papua New Guinea; introduced Madagascar, Reunion \& Mauritius.

Note - As discussed by Nielsen (1980: 349) the entities described as $A$. rugata and $A$. concinna have at times been treated as separate species or as varieties of the one species, but he considered that they are conspecific and correctly applied the name $A$. concinna to the combined entity. Under Senegalia, however, the correct name for this entity is S. rugata. It is regrettable that the well-known epithet concinna must be 
replaced, however, it may possibly be reinstated in the future if studies show that it is best to recognize two taxa for what is here called S. rugata.

21. Senegalia sulitii (I.C.Nielsen) Maslin, Seigler \& Ebinger, comb. nov.

Acacia sulitii I.C.Nielsen, Opera Bot. 81 (1985) 24, f. 10 (6-10). - Type: Sulit PNH 6082 (holo L), Philippines, Samar, Loquilocon, Wright, banks of Ulut River, 100 m, 29 May 1948.

Distribution - Philippines, Indonesia (Sulawesi).

22. Senegalia tawitawiensis (I.C.Nielsen) Maslin, Seigler \& Ebinger, comb. nov.

Acacia tawitawiensis I.C.Nielsen, Opera Bot. 81 (1985) 22, f. 10 (1-5). Type: Ramos \& Edaño BS 44167 (holo NY; iso A, BM, BO, SING), Philippines, Sulu Province, Tawitawi, July-Aug. 1924

Distribution — Philippines (Tawitawi).

23. Senegalia teniana (Harms) Maslin, Seigler \& Ebinger, comb. nov.

Acacia teniana Harms, Repert. Spec. Nov. Regni Veg. 17 (1921) 133. - Type citation: Simeon Ten. 349, 113, "Yunnan: Pe yen tsin"; S. Tenin (Herb. Haun) "San ly tsin Kouty (23.IV.1919)".

Distribution - China (Sichuan, Yunnan).

24. Senegalia thailandica (I.C.Nielsen) Maslin, Seigler \& Ebinger, comb. nov.

Acacia thailandica I.C.Nielsen, Adansonia sér. 2, 19 (1980) 356, pl. 1. — Type: Put 2537 (holo K; iso C, P), Central Thailand (Ang Thong).

Distribution - Thailand, Cambodia.

25. Senegalia tonkinensis (I.C.Nielsen) Maslin, Seigler \& Ebinger, comb. nov.

Acacia tonkinensis I.C.Nielsen, Adansonia sér. 2, 19 (1980) 358, pl. 2. Type: Wilson 2715 (holo K), Vietnam (Lao Cai), Aug. 1899.

Distribution - China (Yunnan), Laos, Vietnam (northern).

26. Senegalia torta (Roxb.) Maslin, Seigler \& Ebinger, comb. nov.

Mimosa torta Roxb., FI. Ind., ed. 2, 2 (1832) 566. - Acacia torta (Roxb.) Craib (1915) 410. - Type: Roxburgh drawing n. 1865 (K)

Distribution - Pakistan, India, Thailand.

Note — Nielsen (1985b: 166), Sanjappa (1992: 44), Kumar \& Sane (2003: 99) and Roskov et al. (2005) recognize Acacia torta as a distinct species, but Chakrabarty \& Gangopadhyay (1996: 604, 606) consider it conspecific with $A$. (Senegalia) caesia.

\section{Senegalia verheijenii (I.C.Nielsen) Maslin, Seigler \&} Ebinger, comb. nov.

Acacia verheijenii I.C.Nielsen, Opera Bot. 81 (1985) 16, f. 6 (1-6). - Type: Verheijen 3504 (holo L), Lesser Sunda Islands, Flores, between N. Lanang and T. Golo, 7 Mar. 1974

Distribution — Indonesia (Flores).

28. Senegalia vietnamensis (I.C.Nielsen) Maslin, Seigler \& Ebinger, comb. nov.

Acacia vietnamensis I.C.Nielsen, Adansonia sér. 2, 19 (1980) 360, pl. 3. — Type: Poilane 19678 (holo K; iso P), Southern Vietnam (Bien Hoa: Dinh-Quan), 13 Oct. 1931.
Distribution - China (Guangxi, Guangdong, Hainan, Hunan, Jiangxi, Zhejiang, Yunnan), Laos, Vietnam.

29. Senegalia yunnanensis (Franch.) Maslin, Seigler \& Ebinger, comb. nov.

Acacia yunnanensis Franch., PI. Delavay. (1890) 193. - Type citation: Delavay 2555 "Yun-nan, in collibus calcareis et silvulis ad Che-tong, prope Tapin-tze; fl. 13 Maj. 1886".

Distribution — China (Yunnan, Sichuan).

\section{Vachellia Wight \& Arn.}

(syn. former Acacia Mill. subg. Acacia)

30. Vachellia harmandiana (Pierre) Maslin, Seigler \& Ebinger, comb. nov.

Pithecolobium? harmandianum Pierre, Fl. Forest. Cochinch. 5 (1899) t. 394A - Acacia harmandiana (Pierre) Gagnep. (1911) 115. — Lectotype: Pierre 5982 “Harmand 77 \& 125” (P; iso E, K, Nielsen 1980: 345), Laos, Dec. 1875

Distribution - Thailand, Laos, Cambodia, Vietnam.

31. Vachellia inopinata (Prain) Maslin, Seigler \& Ebinger, comb. nov.

Acacia inopinata Prain, J. Asiat. Soc. Bengal, Pt. 2, Nat. Hist. 66 (1897) 507 - Type citation: King's Collector, "Shan Hills".

Distribution - Myanmar (Burma).

Note - Despite no formal taxonomic treatment being presented by Nielsen (1981: 42; 1985b: 157) he clearly accepted Acacia inopinata by including it in his key; the provenance was given as Burma. Thus, A. inopinata was out of geographic scope for Nielsen's treatment, but not for his key. This species is also accepted by Kumar \& Sane (2003: 87) and Roskov et al. (2005).

32. Vachellia kingii (Prain) Maslin, Seigler \& Ebinger, comb. nov.

Acacia kingii Prain, J. Asiat. Soc. Bengal, Pt. 2, Nat. Hist. 66 (1897) 506. Type citation: King's Collector, "Shan Hills".

Distribution - Myanmar (Burma).

Note - Despite no formal taxonomic treatment being presented by Nielsen (1981: 42; 1985b: 157) he clearly accepted Acacia kingii by including it in his key; the provenance was given as Burma. Thus, A. kingii was out of geographic scope for Nielsen's treatment, but not for his key. This species is also accepted by Kumar \& Sane (2003: 87) and Roskov et al. (2005).

33. Vachellia leucophloea (Roxb.) Maslin, Seigler \& Ebinger, comb. nov.

Mimosa leucophloea Roxb., PI. Coromandel 2 (1800) 27, t. 150. - Acacia leucophloea (Roxb.) Willd. (1806) 1083. - Lectotype: Roxburgh, PI. Corom. 2 (1800) t. 150; [icon] (Nielsen 1980: 345).

Distribution — Pakistan, India, Sri Lanka, Myanmar (Burma), Thailand, Vietnam (southern), Malaysia, Indonesia (Java, Bali), East Timor; introduced in Trinidad and Mauritius.

Note - Varieties are recognized for this species in ILDIS (Roskov et al. 2005), based on Kumar \& Sane (2003: 89), therefore, combinations in Vachellia are provided here. However, it is noted that in the latter publication the authors' note that the variety is "not maintained in recent literature", citing Chakrabarty \& Gangopadhyay (1996) as their validating reference. 


\section{a. var. leucophloea}

Acacia leucophloea var. leucophloea (Miquel 1855: 9).

Distribution — Pakistan, India, Sri Lanka, Myanmar (Burma).

b. var. microcephala (Kurz) Maslin, Seigler \& Ebinger, comb. nov.

Acacia leucophloea var. microcephala Kurz, J. Asiat. Soc. Bengal, Pt. 2, Nat. Hist. 45 (1877) 297. - Type: None cited in protologue.

Distribution - Myanmar (Burma).

Note - This variety is recognized by Kumar \& Sane (2003, who note that it is not maintained in current literature) and was subsequently listed in Roskov et al. (2005). It was not included in any of Nielsen's publications of Southeast Asian acacias, but this is perhaps not surprising because Myanmar was beyond the geographic scope of Nielsen's work.

\section{Vachellia myaingii (Lace) Maslin, Seigler \& Ebinger, comb. nov.}

Acacia myaingii Lace, Bull. Misc. Inform. Kew (1915) 114, as 'myaingii'. Type citation: Maung Tha Myaing 238 (quoad spec. florifera), "Burma: Meiktila district, Menyo Reserve, 200 m"; English 21, "Wundwin Hill".

Distribution - Myanmar (Burma) (Kress et al. 2003: 299).

Note - Despite no formal taxonomic treatment being presented by Nielsen (1981: 42; 1985b: 157) he clearly accepted Acacia myaingii by including it in his key; the provenance was given as Burma. Thus, $A$. myaingii was out of geographic scope for Nielsen's treatment, but not for his key. The species is also recognized in Kress et al. (2003) but is not included in Lock \& Heald (1994), Kumar \& Sane (2003) or Roskov et al. (2005).

\section{Vachellia siamensis (Craib) Maslin, Seigler \& Ebinger, comb. nov.}

Nimiria siamensis Craib, Bull. Misc. Inform. Kew (1927) 393. - Acacia craibii I.C.Nielsen (1980) 344. - Type: Kerr 10180 (holo K; iso ABD, BM), Thailand, Kanchanaburi, Si Sawat, 150 m, open deciduous forest.

Distribution - Thailand.

Note - In Acacia the correct name for this species is A. craibii because Nielsen (1980) could not adopt the epithet 'siamensis' as the combination was already occupied by A. siamensis Craib (see under V. harmandiana below). However, in Vachellia the epithet is not pre-occupied and therefore the correct name for the species is $V$. siamensis.

\section{Vachellia tomentosa (Rottler) Maslin, Seigler \& Ebinger,} comb. nov.

Mimosa tomentosa Rottler, Ges. Naturf. Freunde Berlin Neue Schriften 4 (1803) 208. - Type citation: "Marmelon [Tharangambadi (formerly Tranquebar), Tamil Nadu, SE India]. Octobr. 16. 1799."

Acacia tomentosa Willd. (1806) 1087. - Type: Rottler 784 (holo B-W) (sphalm. Klein s.n., Nielsen 1981: 50), India, Madras, Marmelon, 16 Oct. 1799 .

Distribution — India, Sri Lanka, Bangladesh, Myanmar (Burma), Thailand, Vietnam, Indonesia (Java, Sumba, Sumbawa, Sulawesi).

Note - This name is presumably homotypic with Mimosa tomentosa Rottler.

\section{DOUBTFUL NAMES}

Acacia brunnescens C.E.Parkinson, Bull. Misc. Inform. Kew (1932) 103. - Type: C.E. Parkinson s.n., Pegu Yoma, Insein District, Pyinmadaw, flowers Dec.

The taxonomic status of this entity requires further study. It was provisionally treated as conspecific with $A$. megaladena by Nielsen (1980: 350; 1981: 72), and although recognized as a Myanmar (Burma) endemic by Kress et al. (2003: 299) the name does not appear in Lock \& Heald (1994), Kumar \& Sane (2003) or Roskov et al. (2005).

\section{Acacia microcephala Graham}

Kress et al. (2003: 299) list this name for an entity from Myanmar, citing its distribution and common names within that country, and describing the plant simply as a 'Tree'. The name A. microcephala Graham first appeared in Wallich Num. List [Wallich] n. 5263 (1831-1832). Names on this list are regarded being effectively published but are to be treated as nomina nuda unless a subsequent combination cites (or infers) a basionym, in which case they are considered to be validly published (Forman 1997). We consider the Kress et al. (2003) listing of $A$. microcephala Graham as a nomen nudum. Besides, even if it were regarded as having validly published it would be a nom. illeg. because it would be a later homonym on account of $A$. microcephala Macfadyen (1837). The taxon to which Kress et al. applied the name $A$. microcephala is perhaps $A$. leucophloea var. microcephala Grah. ex Baker in Hook.f., FI. Brit. India 2 (1878) 294; the latter name is possibly an isonym of $A$. leucophloea var. microcephala Kurz (1877: 297) which is the basionym of Vachellia leucophloea var. microcephala (see above).

\section{Acacia obcordata Hemsl.}

This name appears in Kress et al. (2003: 300) who say that it is "Reported from Myanmar"; the only other information provided is that the plant is described as a "Small tree". We have not been able to locate this name in any source available to us and it is therefore considered nomen nudum, but the taxon to which this name might apply is unknown.

Acacia pseudoarabica Blume ex Miq., FI. Ind. Bat. 1 (1855) 8. - Type: ? Zippelius (Verdcourt 1979: 172-173), Nov. Guinea.

According to Verdcourt (1979) this name is based on a cultivated specimen of $A$. (Vachellia) nilotica (L.) Willd. ex Delile.

Acknowledgements We are very grateful to Paul Wilson, W.A. Herbarium, for advice on many nomenclatural matters included in this paper. The taxonomic conclusions, however, are ours alone. Libing Zhang, Missouri Botanical Garden, is thanked for providing translation from Chinese of the type information for Acacia delavayi var. kunmingensis and Acacia pruinescens var. luchunensis. Finally, we wish to sincerely thank the two anonymous reviewers whose detailed and constructive comments have improved this paper.

\section{REFERENCES}

Banfi E, Galasso G. 2008. New combinations in Vachellia Wight \& Arn., formerly Acacia Mill. ss. (Fabaceae). Della Sociieta Italiana di Scienze Naturali 149: 149-150.

Bentham G. 1842. Notes on Mimoseae, with a synopsis of species. London Journal of Botany 1: 318-528.

Britton NL, Rose JN. 1928. North American Flora. Mimosaceae 23: 1-194. New York Botanical Gardens, New York.

Candolle AP. 1825. Prodromus Systematis Naturalis Regni Vegetabilis 2: 1-644. Treuttel \& Würtz, Paris 
Chakrabarty T, Gangopadhyay M. 1996. The genus Acacia P. Miller (Leguminosae: Mimosoideae) in India. Journal of Economic and Taxonomic Botany 20: 599-633.

Clarke HD, Seigler DS, Ebinger J. 2009. Taxonomic revision of the Vachellia acuifera species group (Fabaceae: Mimosoideae) in the Caribbean. Systematic Botany 34: 84-101.

Craib WG. 1915. Mimosa caesia and M. intsia. Bulletin of Miscellaneous Information: 407-410. Royal Botanic Gardens, Kew.

Forman L. 1997. The Wallich Herbarium and Catalogue. Unpublished information sheet. Royal Botanic Gardens, Kew, London.

Gagnepain F. 1911. Mimosées nouvelles. Notulae Systematicae 2, 4: 113120. Geuthner, Paris.

Glass CE, Seigler DS. 2006. A new combination in Senegalia and typification of six New World Acacia names. Taxon 55, 4: 993-995.

Hurter PJH, Mabberley DJ. 2008. New names used in this book. In: Mabberley DJ, Mabberley's plant-book: 1021. Cambridge University Press, Cambridge, England.

Kodela PG, Wilson PG. 2006. New combinations in the genus Vachellia (Fabaceae: Mimosoideae) from Australia. Telopea 11: 233-244.

Kress WJ, De Filipps R, Farr E, Yin Yin Kyi D. 2003. A checklist of the trees, shrubs, herbs and climbers of Myanmar. Contributions from the United States National Herbarium 45: 1-590.

Kumar S, Sane PV. 2003. Legumes of South Asia. A check-list: 1-536. Royal Botanic Gardens, Kew, London.

Kurz WS. 1877. Contributions towards a knowledge of the Burmese Flora. Journal of the Asiatic Society of Bengal, Part 2, Natural History 46: 204-310.

Kyalangalilwa B, Boatwright JS, Daru BH, Maurin O, Van der Bank M. 2013. Phylogenetic position and revised classification of Acacia s.I. (Fabaceae: Mimosoideae) in Africa, including new combinations in Vachellia and Senegalia. Botanical Journal of the Linnean Society. doi: 10.1111/boj.12047.

Lock JM, Heald J. 1994. Legumes of Indo-China. A check-list. Royal Botanic Gardens, Kew, London.

Macfadyen J. 1837. The flora of Jamaica 1: 316. Longman et al., London; Black \& Black, Edinburgh; Smith \& Son, Glasgow.

Maslin BR. 2012. New combinations in Senegalia (Fabaceae: Mimosoideae) for Australia. Nuytsia 22: 465-468.

McNeill J, Barrie FR, Buck WR, Demoulin V, Greuter W, et al. 2012. International Code of Nomenclature for algae, fungi, and plants (Melbourne Code). Regnum Vegetabile 154. Published for IAPT by ARG Gantner Verlag, Ruggell, Liechtenstein.
McNeill J, Turland NJ. 2011. Major changes to the Code of Nomenclature Melbourne, July 2011. Taxon 60: 1495-1497.

Miller JT, Seigler DS. 2012. Evolutionary and taxonomic relationships of Acacia s.l. (Leguminosae: Mimosoideae). Australian Systematic Botany 25: 217-224.

Miquel FAW. 1855. Flora van Nederlandsch Indië. Van der Post, Amsterdam. Nielsen I. 1980. Notes on Indo-Chinese Mimosaceae. Adansonia sér. 2, 19: 339-363.

Nielsen I. 1981. Legumineuses-Mimosoidées. Flore du Cambodge, du Laos et du Viet-Nam 19: 3-159. Muséum National d'Histoire Naturelle, Paris.

Nielsen I. 1985a. The Malesian species of Acacia and Albizia (Leguminosae-Mimosoideae). Opera Botanica 81: 1-50.

Nielsen I. 1985b. Leguminosae-Mimosoideae. In: Smitinand T, Larsen K (eds), Flora of Thailand 4: 131-222. Forest Herbarium, Royal Forest Department, Bangkok.

Nielsen I. 1992. Mimosaceae (Leguminosae-Mimosoideae). Flora Malesiana, Ser. I, 11: 1-226. Rijksherbarium / Hortus Botanicus, Leiden.

Roskov YR, Bisby FA, Zarucchi JL, Schrire BD, White RJ (eds). 2005. ILDIS, world database of Legumes: draft checklist, Version 10. CD ROM. Reading, UK. http://www.ildis.org/.

Sanjappa M. 1992. Legumes of India. Bishen Singh Mahendra Pal Singh, Dehradun, India.

Seigler DS, Ebinger JE. 2005. New combinations in the genus Vachellia (Fabaceae: Mimosoideae) from the New World. Phytologia 87: 139-178. Seigler DS, Ebinger JE. 2010. New combinations in Senegalia and Vachellia (Fabaceae: Mimosoideae). Phytologia 92: 90-93.

Sun H, Chen J. 1990. Taxonomy, distribution and possible florestic origin of the genus Acacia from China. Acta Botanica Yunnanica 12: 255-268.

Turland NJ, Jarvis CE. 1997. Typification of Linnean specific and varietal names in the Leguminosae (Fabaceae). Taxon 46: 457-485.

Turner LM. 1997, '1995'. A catalogue of the vascular plants of Malaya. Gardens' Bulletin Singapore 47, 1: 1-346.

Van Steenis CGGJ. 1948. Introduction. Flora Malesiana, Ser. I, 4: v-xii. Noordhoff-Kolff, Batavia.

Verdcourt B. 1979. A manual of New Guinea legumes. Botany Bulletin 11: 1-645. Office of Forests Division of Botany, Lae.

Willdenow CL. 1806. Species Plantarum edn 4, 4 (2): 633-1157. Nauk, Berlin. Wu De-Lin, Nielsen IC. 2010. Acacieae. In: Wu ZY, Raven PH, Hong DY (eds), Flora of China 10: 55-59. Science Press, Beijing \& Missouri Botanical Garden Press, St. Louis. 